\title{
A Physical Model of the Effect of a Shallow Weak Layer on Strong Ground Motion for Strike-Slip Ruptures
}

\author{
James N. Brune and Abdolrasool Anooshehpoor \\ Seismological Laboratory, University of Nevada-Reno
}

\begin{abstract}
We report results of foam-rubber modeling of the effect of a shallow weak layer on ground motion from strike-slip ruptures. Computer modeling of strong ground motion from strikeslip earthquakes has involved somewhat arbitrary assumptions about the nature of slip along the shallow part of the fault (e.g., fixing the slip to be zero along the upper 2 kilometers of the fault plane) in order to match certain strong motion accelerograms. Most modeling studies of earthquake strong ground motion have used what is termed kinematic dislocation modeling. In kinematic modeling the time function for slip on the fault is prescribed, and the response of the layered medium is calculated. Unfortunately, there is no guarantee that the model and the prescribed slip are physically reasonable unless the true nature of the medium and its motions are known ahead of time.
\end{abstract}

There is good reason to believe that in many cases faults are weak along the upper few kilometers of the fault zone and may not be able to maintain high levels of shear strain required for high dynamic energy release during earthquakes. Physical models of faulting, as distinct from numerical or mathematical models, are guaranteed to obey static and dynamic mechanical laws. Foam-rubber modeling studies have been reported in a number of publications. The object of this paper is to present results of physical modeling using a shallow 
weak layer, in order to verify the physical basis for assuming a long rise time and a reduced high frequency pulse for the slip on the shallow part of faults. It appears a 2-kilometer deep, weak zone along strike-slip faults could indeed reduce the high frequency energy radiated from shallow slip, and that this effect can best be represented by superimposing a small amplitude, short rise-time pulse at the onset of a much longer rise-time slip. A reak zone was modeled by inserting weak plastic layers of a few inches in thickness into the foam rubber model. For the $15 \mathrm{~cm}$ weak zone the average pulse is reduced by a factor of 0.46 . The factor for the $20 \mathrm{~cm}$ case reduction is 0.11 . For the $30 \mathrm{~cm}$ case it is 0.045 . From these results we can see that, the thicker the weak layer, the more difficult it is for a short rise-time acceleration pulse to push its way through the weak layer to the surface. This is thus an approximate justification for reducing the high frequency radiation from shallower parts of strike-slip faults if it is known that the shallow part of the fault is weak or has not stored up shear stress.

\section{Introduction}

Modeling of strong ground motion from strike-slip earthquakes has involved somewhat arbitrary assumptions that the slip along the shallow part of the fault was essentially zero, in order to match some strong motion accelerograms (Campbell, 1997; Marone and Scholz, 1988). It was realized that this was an ad hoc and unsatisfactory situation from a physical point of view, since it is well known that the fault slip at the surface is commonly of the same magnitude as slip at depth.

It is generally accepted that the static normal and shear stresses along a vertical strikeslip fault must approach zero if the surface consists of incompetent sediments, because such materials could not maintain permanent stresses. Even in competent rocks the shear stresses in bore holes are commonly observed to approach zero near the surface in strike-slip faulting regimes. Since the shear stresses are approximately related to the normal stresses by the coefficient of friction, $\mu$, and since the weight of the overlying rocks approaches zero, the shear 
stresses held by rock weight should also approach zero. Of course if tectonic normal forces are present (Zoback et al., 1987), then normal shear stresses near the surface in competent rocks could theoretically be quite high. In most developed fault zones the fault zone at shallow depths is a layer of relatively incompetent fault gouge, which would not be expected to be able to maintain large shear strains over inter-seismic time periods. In extensional tectonic regimes, such as Basin and Range Province of the western U.S., the normal stress must approach zero at the surface because the lithostatic stress is zero and the horizontal stress is extensional. Most measurements of shear stress in the western United States show values at zero near the surface (Zoback and Zoback, 1980). Therefore there is good reason to believe that faults are often weak along the upper few kilometers of the fault zone, and may not be able to maintain high levels of shear strain required for high dynamic energy release. This in turn might be the explanation for the presumed requirement to reduce dynamic slip along the shallow parts of faults in strong-motion modeling.

However, given the fact that the slip is known not to be zero at the surface, but comparable to slip at depth, it seems that, rather than locking the fault at shallow depths, a more reasonable solution would be to decrease the fault slip velocity (lengthen the rise-time of the fault slip), thus decreasing the amount of high frequency energy radiated, but still allow slow slip to continue to match the final fault offset.

The object of this paper is to present results of physical modeling using a shallow weak layer, to suggest physical basis for assuming a long rise-time and a reduced high frequency generation on the shallow part of faults.

\section{Rationale for Foam-rubber Modeling}

Physical models of faulting, as distinct from numerical or mathematical models, are guaranteed to obey static and dynamic mechanical laws. Thus these models can be used to gain insight into the physical processes involved. Of course there are inherent problems of scaling 
and matching of physical conditions of laboratory physical models with the real Earth. Such models can nonetheless provide important constraints on numerical and theoretical models, for example, by modeling dynamic geometrical effects on fault slip.

Foam-rubber is very flexible, i.e., it has a low rigidity, making it is easy to produce large strains and particle motions. Since foam-rubber is light-weight, relatively large models can be constructed, enabling the scale of dynamic phenomena to be enlarged. This allows dynamic features to be more easily observed and recorded using relatively simple electronic devices, such as tiny accelerometers and position sensing devices. Foam-rubber models automatically assure that motions are physically realistic (no singularities or unreasonably specified slips).

Great effort has been expended in rock mechanics laboratories to determine the properties of slip along interfaces between small blocks (centimeters to meters) of rock in hopes that these results could somehow be scaled up to the dimensions of rocks involved in real earthquakes (tens of kilometers or more). However, such scaling has never been justified in the literature. There are two dynamic scaling considerations not satisfied by ordinary rock mechanics experiments which are satisfied by the foam-rubber model. First, the stressing apparatus for the foam model has effectively infinite rigidity compared to the rigidity of the model, assuming that the dynamics of the model are not influenced by interaction with the stressing apparatus. We have verified this by placing a small accelerometer on the piston that stresses the model. No measurable motion of the piston (no interaction) was found at the time of a stick-slip event. Secondly, the overall dimensions of the foam rubber model are large compared to the dimension of dynamic slip pulse which propagates along the interface between the two blocks. This allows the slip pulse to propagate predominantly under the influence of conditions local to the slip pulse itself, with minimized effects of the boundaries of the model and the stressing apparatus. This obviously corresponds better to the conditions in the earth, for which the length of slip pulse is small compared to the dimensions of the fault (Heaton, 1990). Foam rubber has a Poisson's ratio of about 0.3 , close to that for rocks, and thus satisfies one of the dimensionless scaling requirements, the ratio of $P$-wave to $S$-wave 
velocities.

Some of the major limitations of foam-rubber modeling include:

1. Intrinsic $Q$ is low (on the order of 10) and cannot be controlled. This constrains the usefulness of foam-rubber modeling to wave propagation distances which are not too large compared to the wavelengths involved. Thus it is most useful to gain insight into near-source phenomena. An ancillary advantage of the low $Q$ is that there is relatively little energy scattered back from the model's distant sides, which could interfere with the dynamics.

2. The fault surface friction conditions are difficult to control. The lattice of foam-rubber vesicles produces extreme roughness on a small scale (of the order of a millimeter). The coefficient of friction is on the order of 10 , whereas that for rocks is of the order of 0.5. Thus to produce fault slip, the strains must be very large, of the order of $10^{-2}$, whereas in the earth the corresponding strains are of the order of $10^{-4}$. As long as strains are approximately linear, the difference can be corrected for. However, in both the foam-rubber and the real earth, the fault behavior is probably not linear on the fault trace, and there is no guarantee that the non-linearities in the foam model correspond to those in the real earth. (A similar problem is common to kinematic modeling as well.)

\section{Description of Model}

The model consists of two large blocks of foam rubber $(1 \mathrm{~m} \times 2 \mathrm{~m} \times 2.5 \mathrm{~m})$, one driven horizontally over the other by a hydraulic piston (Figure 1). The lower block is securely glued to a plywood sheet which is in turn anchored to the concrete floor. The upper block and the attached rigid frame are supported by four steel pipes equipped with scaffolding jacks and guiding rollers at each corner. Thin sheets of plywood are glued to the front and the back of each block. These sheet, $\mathrm{s}$ are free to rotate about the hinges connecting them to the two horizontal plywood sheets attached to the top of the upper block and the bottom of the 
lower block. When the model is under shear force, these sheets prevent the foam blocks from tearing off the horizontal plywood plates.

Normal force at the contact (fault) is provided by some fraction of the weight of the upper block $(300 \mathrm{~kg})$ and is varied by lowering or raising the jacks. Normal force is measured by subtracting the force exerted on the jacks from the total weight of the upper block. Shear force is provided by a hydraulic piston that is placed between a concrete wall and the upper block's frame; the rollers guiding the moving block ensure a pure shear motion.

As the upper block is forced to slide over the lower block, the strain in the blocks increases until the stress at the interface exceeds the frictional resistance and a stick-slip event occurs over the whole boundary (fault plane). These major events correspond to "characteristic" events for the system, perhaps analogous to "characteristic earthquakes" in the earth (Schwartz and Coppersmith, 1984). Successive "characteristic" events usually cause about the same amount of average slip $(1 \mathrm{~cm})$ between the blocks; but the pattern of slip can vary markedly, with the rupture initiating at different points and propagating in different directions. If the driving displacement is steady, the characteristic events repeat more or less regularly until the upper block has slipped about 30 centimeters, corresponding to about 30 characteristic events with some additional smaller events. At this point, the hydraulic piston is fully extended and one experimental run is complete. The stress is removed and the upper block lifted and moved back to the starting position for repeat of the procedure. Near the point of shear failure events can be caused to nucleate at different points by slightly raising one or more of the jacks.

\section{Instrumentation}

\section{Position-sensing Detectors}

Displacement at the foam surface is measured by a telescopic, 2-axis, position-sensing detector, which is focused on a small light emitting diode (LED) embedded in the foam. The Dual 
Axis Super Linear Position Sensor (DLS10, manufactured by the United Detector Technology Sensors, Inc.), is a square of photovoltaic material, $1 \mathrm{~cm}$ on a side. The sensor locates the centroid of a light spot (image of the embedded LED) projected upon it, and provides continuous output as the light spot moves from the null point to either direction along each of the two perpendicular axes. The output of the position-sensing detector depends on the location as well as the intensity of the bright spot. Therefore, it is necessary to calibrate detectors before and after each experimental run. (The position detectors have a built-in calibrating mechanism.) The resolution of the DLS10 sensors is limited only by the intensity of the light source and the signal resolving circuitry. In our experiments, the resolution is better than $0.01 \mathrm{~cm}$.

\section{Ultra-light Accelerometers}

Due to foam rubber's low density and high elasticity, particle accelerations in a stressed foam rubber model of earthquakes can exceed several hundred $g$ (the acceleration due to gravity). Slips of the order of $1 \mathrm{~cm}$ can take place in a few milliseconds (ms), resulting in very large accelerations at high frequencies. In order to measure these accelerations, accelerometers with a high dynamic range and low mass (to minimize the mass loading effects) are needed. We have 22 state-of-the-art, ultra-light ENDEVCO Model 25A accelerometers. The Model 25A, with a mass of $0.2 \mathrm{gm}$ and a dynamic range of $\pm 1000 \mathrm{~g}$, is the world's smallest piezoelectric accelerometer. In order to further reduce the mass loading effects, each accelerometer is mounted on a styrofoam disk, $3.8 \mathrm{~cm}$ in diameter, before inserting them in the foam; the $3 \mathrm{~mm}$ thick styrofam disk (with the same density as the foam rubber used in the model, but far more rigid) distributes the accelerometer's mass over a larger area (about 50 times larger).

\section{Data Acquisition System}

The data acquisition system consists of a $486 \mathrm{PC}$ with a $330 \mathrm{kHz}$ analog-to-digital board. Particle motions during stick-slip events at 16 sites were digitized at the rate of 5000 samples- 
per-second and recorded on the PC.

\section{Experimental Procedures}

' In this study, we are modeling strike slip motion, so the motion sensors are installed along the right side of the interface looking from the piston in the direction of motion of the upper block (in effect the model is like a strike-slip fault rotated 90 degrees on its side). We had verified in previous experiments that the particle motions are approximately symmetrical on opposite sides of the fault in the model with no plastic strip inserted. Thus the main features of the distribution of particle motion could be determined by recording on only one side of the fault.

Figure 2 shows the location of fourteen miniature accelerometers that are embedded in the lower block (indicated by numbers 1-13, and 15) and one in the upper block (number 14) near the fault surface. Accelerometer 14 in the upper block is approximately located above accelerometer 15 in the lower block (the relative position changes by about \pm 10 centimeters as the upper block is pushed over the lower one). A light emitting diode inserted at site 16 (on the surface) is used to monitor the slip during each stick-slip event and cross check the accuracy of double-integration process in calculating displacements from accelerations. Sensors at all 16 sites measure particle motions on the fault plane parallel to the direction of external shear force (provided by the hydraulic piston).

In order to determine the effect of a shallow weak layer we carried out several experiments with strips of low-friction plastic of different widths inserted along the edge of the model corresponding to the surface of the earth. The measured quasi-static friction of the plastic is about 0.17 of the rupture strength of the foam block interface. The widths of the strips were 0 (no strip), $7.5 \mathrm{~cm}, 15 \mathrm{~cm}, 20 \mathrm{~cm}$, and $30 \mathrm{~cm}$ (Figure 2). We perturbed the stresses in the model to cause nucleation of the rupture to occur at depth, and thus caused the rupture front to arrive at the surface with a steep angle of incidence, as is typical of actual strike-slip 
earthquakes.

\section{Data}

The main variable in our experiment is thickness of the weak layer (width of the plastic strip). In order to make clear the effect of the shallow weak layer, we recorded several rupture events without the plastic strip inserted, and several for each of the widths of plastic strip.

\section{Long Rise-time Slip}

In the cases with weak layers, even though the peak accelerations were greatly reduced by the weak layer, the total slip was not. This can easily be seen by comparing the total slips shown on channel 16 , for the cases with and without the weak zones (Figure 3 ). We have also confirmed this fact by integrating the accelerometer channels to get fault displacement. Thus the shallow weak layer reduces the amplitude of the short rise-time pulse, but not the overall slip, which is accomplished by long rise-time slip. Typical rise-times of the displacement time functions shown in the figures are about $50 \mathrm{~ms}$. In general, there is also a small high velocity pulse near the beginning of the ramp, the pulse amplitude depending on the thickness of the weak layer.

\section{Short Rise-time Pulse Propagating Through the Weak Layer}

In most cases with a shallow weak zone, a short rise-time pulse propagates through the weak zone and arrives at the surface, but with reduced amplitude compared to the case with no weak zone. This is illustrated in Figure 3. In order to determine the average effect of the shallow weak layer, we have averaged the accelerations for each case and shown the results in Figure 4 (Since the average amplitude of the dislocation at depth varied for each of theses cases, they were normalized to the amplitude interpolated for a position half way between 
sensor 1 and sensor 2).

From the results in Figure 5 we see that the pulse observed for the $7.5 \mathrm{~cm}$ layer has been reduced by a factor of 0.4 over the average value for the case with no weak zone. For the $15 \mathrm{~cm}$ wide weak zone the average pulse is reduced by a factor of 0.46 . The factor for the $20 \mathrm{~cm}$ case reduction is 0.11 . For the $30 \mathrm{~cm}$ case it is 0.045 . From these results we see that, the thicker the weak layer, the more difficult it is for a short rise-time acceleration pulse to propagate through the weak layer to the surface.

\section{Analysis}

It is clear from the results above that the effect of the shallow weak layer (strip of plastic) is in all cases to reduce the surface acceleration at the surface site 15 relative to the acceleration observed there with no weak layer (and also relative to the accelerations occurring deeper in the model, corresponding to accelerations deeper in the earth). However, in most cases, a small, short rise-time pulse propagates through the weak zone and reaches the surface, although with much reduced amplitude compared to the case without a weak shear zone. If the results above are applicable to real earth situations, kinematic modeling of ground motion using classical dislocation theory should apply some adjustment of the fault slip time function on the shallow part of the fault. In order to apply these results to the real earth, we must consider the typical ratios of the wavelengths involved to the thichness of the weak layer, as well as other factors which might qualify the results.

The physical reason that the shallow weak zone reduces the surface ground accelerations near the fault is that energy is taken out of the dislocation pulse as it propagates through the weak near-surface zone. This in turn is because this zone has no stored up energ. to replace the energy lost by damping and high velocity sliding friction. It is certain that, in many cases in the real earth, a similar situation occurs, especially where there is a thick and deep weak zone of fault gouge near the surface, or a thick layer of incompetent sediments 
which cannot store long term stresses. To scale these results to the real earth, the critical parameter is the ratio of the wavelength, $\lambda$, of the energy involved to the thickness, $h$, of the weak layer. In the model, the predominant period of the peak pulse of energy reaching the surface for the case of no weak layer is about $10 \mathrm{~ms}$. Multiplying by the typical shear wave velocity, $30 \mathrm{~m} / \mathrm{s}$, we estimate a predominant wavelength of about $30 \mathrm{~cm}$. In the case of the $7.5 \mathrm{~cm}$ weak layer, the high frequency pulse for deep nucleation was observed at the surface, reduced by a factor of 0.46 , whereas only very much weaker pulses were observed for the thicker weak layers (A $2 \mathrm{~cm}$ layer would have been essentially ineffective in damping out the sharp acceleration pulse). The critical ratio of $\lambda / h$ for transition of effectiveness in damping out the sharp acceleration pulse is about $1.5(30 / 20)$. In the real earth, peak accelerations are typically caused by energy of about $5 \mathrm{~Hz}$. If the surface velocities are about $3 \mathrm{~km} / \mathrm{s}$, this corresponds to wavelengths of about 600 meters. Thus a weak layer of about $900 \mathrm{~m}$ would be expected to significantly reduce $5 \mathrm{~Hz}$ energy. For $2.5 \mathrm{~Hz}$ energy, the corresponding thickness would be $1.8 \mathrm{~km}$, close to the value suggested by some studies of strong ground motion (about $2 \mathrm{~km}$ ).

Another factor important in scaling is the ratio of the wavelength of the energy involved to the dimension of the dislocation pulse at depth traveling up the fault plane, the so-called Heaton pulse (Heaton, 1990). In previous studies of foam-rubber modeling we have found that the dimension of the dislocation pulse; i.e., the distance between the leading edge of the rupture and the following edge (locking edge) is about $10 \mathrm{~cm}$. (This seems to be primarily controlled by the characteristic roughness dimension of the interface, in the case of foam-rubber, about one $1 \mathrm{~mm}$.) In the real earth, Heaton (1990) has estimated that the typical dislocation width is about 1-5 kilometers (rise-times of about 0.3 to $2.0 \mathrm{~s}$ ). Thus a wavelength of about $2 \mathrm{~km}$ (about $1 \mathrm{~Hz}$ ) in the earth corresponds to about $10 \mathrm{~cm}$ in the foam model. The shear wave velocity in the foam-rubber is about $35 \mathrm{~m} / \mathrm{s}$, so that $10 \mathrm{~cm}$ corresponds to periods of about $3 \mathrm{~ms}$. The acceleration pulses have energy of this frequency and lower. Similar dynamic geometrical effects should apply to higher frequencies also, if they had been present in the upcoming pulse. 
Thus it appears a shallow weak zone along strike-slip faults could indeed reduce the high frequency energy radiated from shallow slip. This effect can best be represented by superimposing a small amplitude, short rise-time pulse at the onset of a much longer rise-time slip. The average rise-times can be estimated from the LED displacement sensor plots in each figure. The average rise-times are about 3 to 5 times longer for the 15-30 cm strips, compared to the case for no weak layer. This is thus an approximate justification for increasing the rise-time in modeling the radiation from shallower parts of strike-slip faults.

\section{Conclusion}

Results of foam-rubber modeling of the effect of a shallow weak layer on ground motion from strike-slip ruptures indicate a strong damping of the surface acceleration by such a weak layer. Since modeling of ground motion from strike-slip earthquakes has involved somewhat arbitrary assumptions about the nature of slip along the shallow part of the fault, there is need for a physical basis to justify and constrain the parameterization. There is good reason to believe that faults are weak along the upper few $\mathrm{km}$ of the fault zone, especially in extensional regions. Results presented here give a physical basis for assuming a long rise-time and a reduced high frequency pulse for the slip on the shallow part of faults. The acceleration pulses observed at the surface are reduced by factors of $0.4,0.46,0.11$, and 0.045 for the $7.5 \mathrm{~cm}, 15 \mathrm{~cm}, 20 \mathrm{~cm}$, and $30 \mathrm{~cm}$ cases, respectively. From these results we can see that, the thicker the weak layer, the more difficult it is for a short rise-time acceleration pulse to propagate its way through the weak layer to the surface. It appears that this effect can best be represented by superimposing a small amplitude, short rise-time pulse at the onset of a much longer rise-time slip. These results give an physical justification for reducing the high frequency radiation from shallower parts of strike-slip faults, at least in extensional regimes. 


\section{Acknowledgmentśs}

This work was supported by the Yucca Mountain Site Characterization Office as part of . the Civilian Radioactive Waste Management Program. This project is managed by the U.S. Department of Energy, Yucca Mountain Site Characterization Project.

\section{References}

Campbell, K. W. (1997), Empirical near-source attenuation relationships for horizontal and vertical components of peak ground acceleration, peak ground velocity, and pseudoabsolute acceleration response spectra, Seism. Res. Letts., vol. 68, no. 1, pp. 154-179.

Heaton, T.H. (1990), Evidence for and implications of self-healing pulses of slip in earthquake rupture. Physics of the Earth and Planetary Interiors, 64, 1-20.

Marone, C. , C. H. Scholz (1988), The depth of seismic faulting and the upper transition from stable to unstable slip regimes, Geophys. Res. Letts., vol. 15, no. 6, pp. 621-624.

Schwartz, D. P. , and K. J. Coppersmith (1984), Fault behavior and characteristic earthquakes: examples from the Wasatch and San Andreas Fault Zones, J. Geophys. Res., $89, \mathrm{B7}, 5681-5698$.

Zoback, M. D. , Zoback, M. L., Mount, V. S., Suppe, J., Eaton, J. P., Healy, M. D., Oppenheimer, D., Reasenberg, P., Jones, L., Raleigh, C. B., Wong, I. G., Scotti, O., and Wentworth, C., (1987), New Evidence on the State of Stress of San Andreas Fault System, Science, 238, 1105-1111.

\section{Figure Captions}

Figure 1 Diagram of foam-rubber model setup for testing the effect of shallow weak layers on strike-slip motion. Dimensions and description are given in the text. 
Figure 2 Diagram showing setup for sensors in the model. Sensors 1 through 12 are embedded in the model about $3 \mathrm{~cm}$ below the fault surface; sensors 13 through 16 are embedded in the model near the edge (corresponding to ground surface in the Earth). All sensors are embedded in the lower block except for sensor 14 which is embedded in the upper block approximately above sensor 15 . Sensors 1 through 15 are accelerometers; sensor 16 is a LED which was used to measure fault slip. Different style dashed lines indicate the location of plastic strips that were used to simulate shallow weak zones of different depths.

Figure 3 An example of acceleration and displacement records for cases of strike-slip motion with no shallow weak layer and with a $20 \mathrm{~cm}$ shallow weak layer. Numbers shown in the upper right corner of each frame correspond to the sensor number in Figure 2. In each column, please note the different beginnings for time axes on acceleration and displacement plots.

Figure 4 Plot of normalized average acceleration as a function of sensor position between sensor 1 (deepest) and sensor 15 (surface) for the cases of no shallow weak layer, and $7.5 \mathrm{~cm}, 15 \mathrm{~cm}, 20 \mathrm{~cm}$, and $30 \mathrm{~cm}$ weak layers (plastic strips), respectively. Curves are normalized to the average acceleration of sensors 1 and 2 at a point halfway between them. 


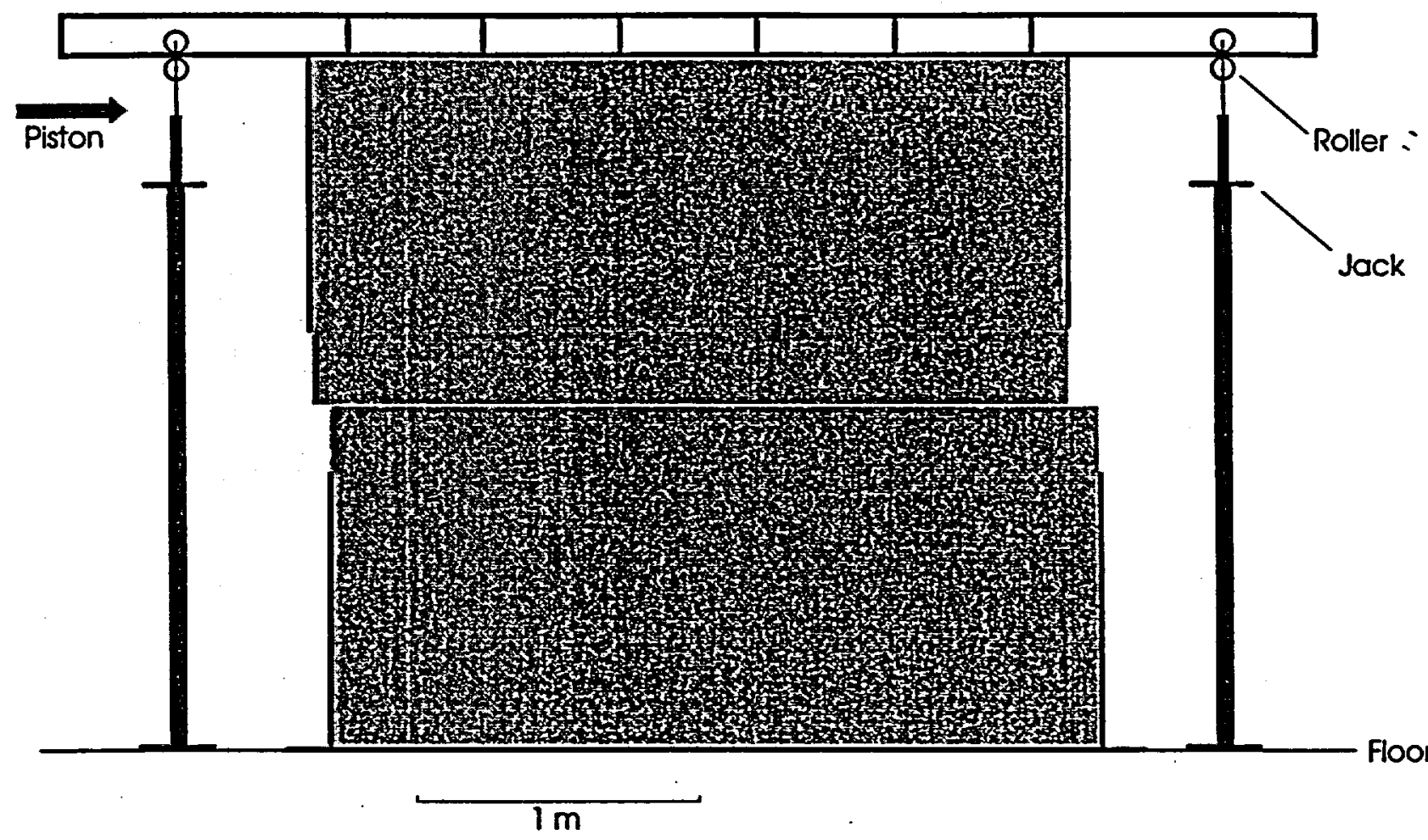

Figure 1 

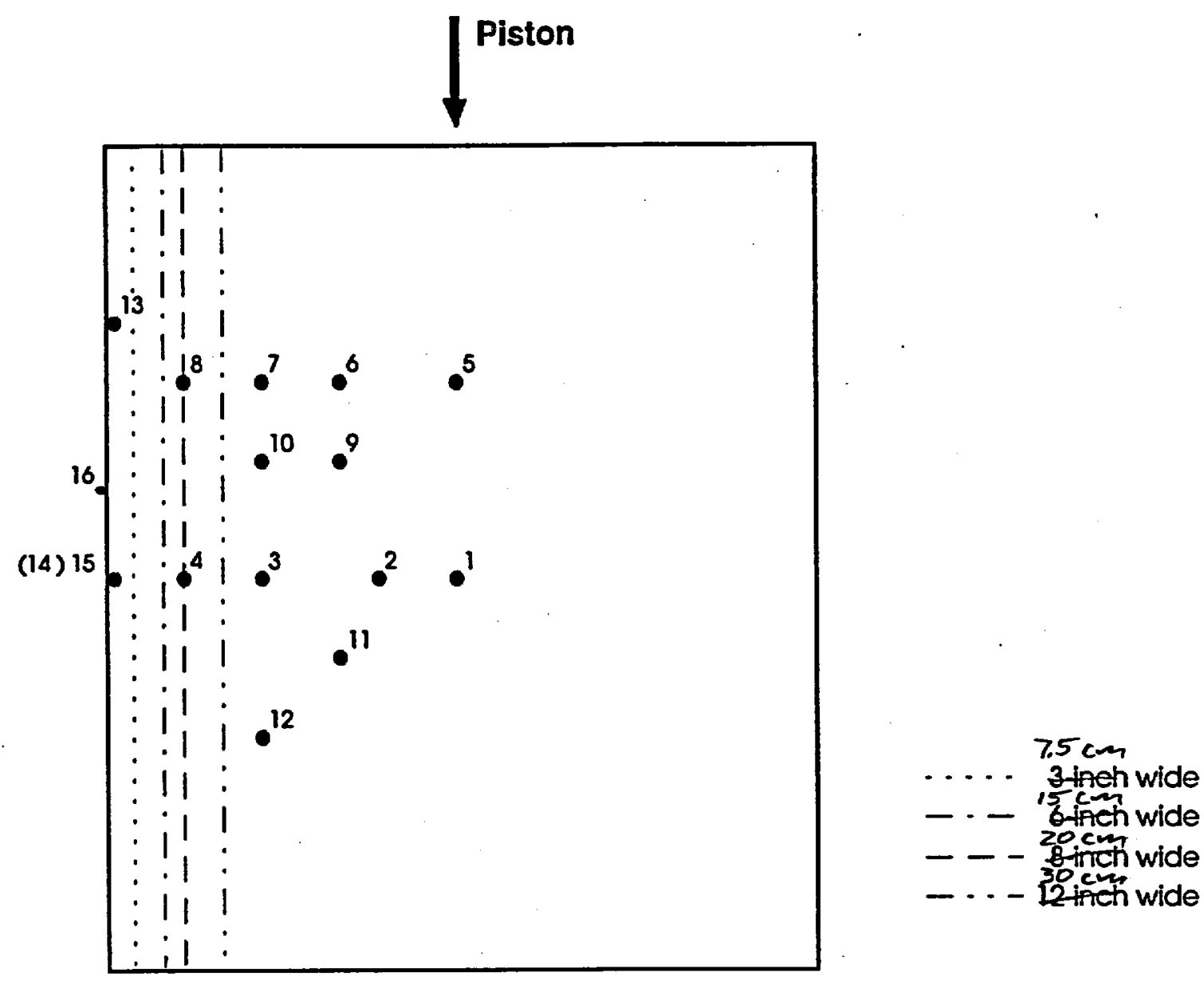

Fault Plane (Lower Block)

Figure 2 
No.Ptutiontrip

$20 \mathrm{~cm}$ Wide Plastic Strip
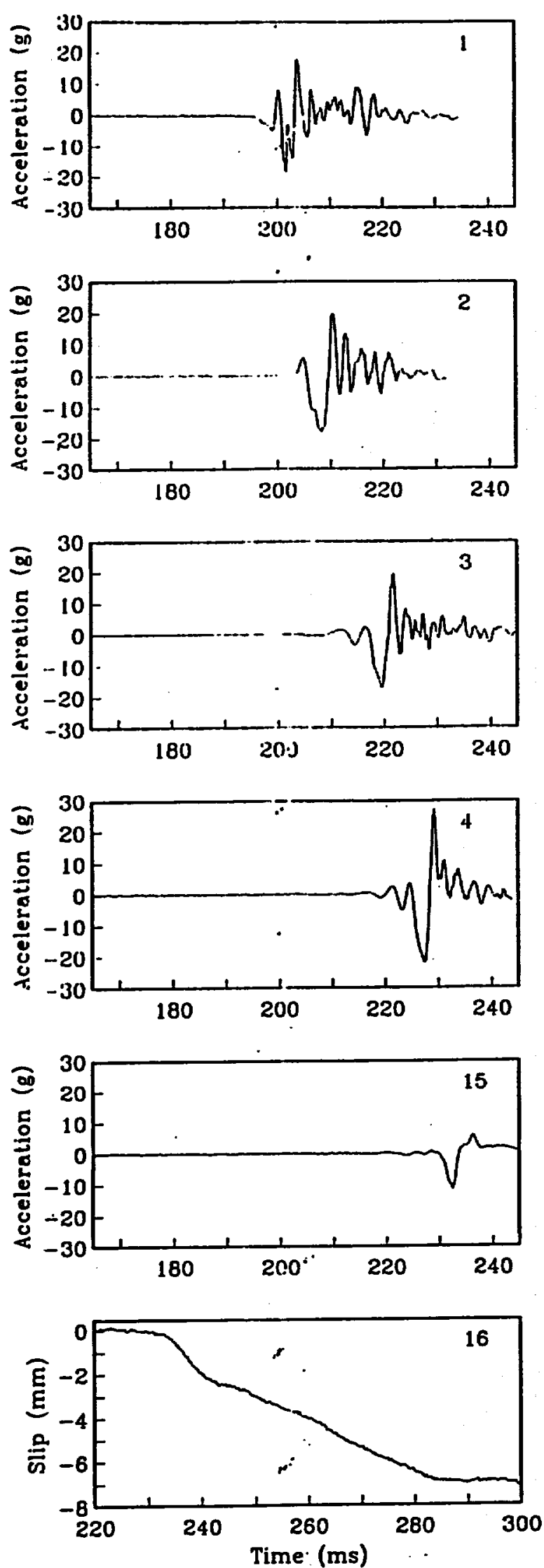

30 em Nide Plastic strip

No Plastic Strip


Figure 3 


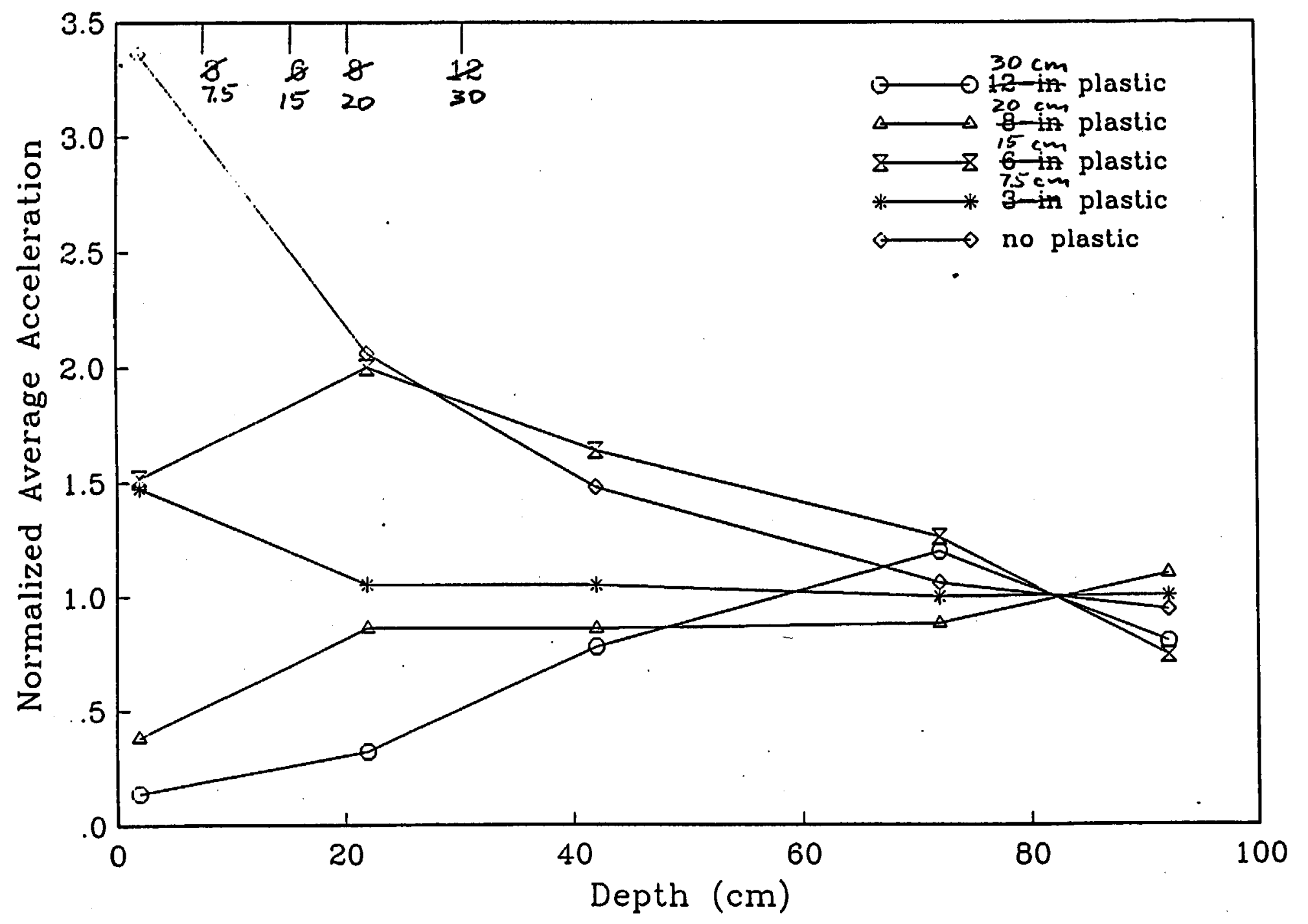

Figure 4 